\title{
Solving the TTC 2013 Flowgraphs Case with FunnyQT
}

\author{
Tassilo Horn \\ horn@uni-koblenz.de \\ Institute for Software Technology, University Koblenz-Landau, Germany
}

\begin{abstract}
FunnyQT is a model querying and model transformation library for the functional Lisp-dialect Clojure providing a rich and efficient querying and transformation API.

This paper describes the FunnyQT solution to the TTC 2013 Flowgraphs Transformation Case. It solves all four tasks, and it has won the best efficiency award for this case.
\end{abstract}

\section{Introduction}

Funny $Q T T^{1}$ is a new model querying and transformation approach which is implemented as an API for the functional, JVM-based Lisp-dialect Clojure. It provides several sub-APIs for implementing different kinds of queries and transformations. For example, there is a model-to-model transformation API, and there is an in-place transformation API for writing programmed graph transformations. FunnyQT currently works natively with EMF and JGraLab models, i.e., it doesn't use some internal model representation, and it can be extended to other modeling frameworks, too.

For solving the tasks of this transformation case 2 . FunnyQT's model transformation API and its polymorphic function API have been used for task 1. Both task 2 and task 3 have been tackled algorithmically using FunnyQT's plain querying and model manipulation APIs. Task 4 has been solved by using FunnyQT's querying API and Clojure metaprogramming.

\section{Solution Description}

Task 1: JaMoPP to StructureGraph. According to the case description [2], the goal of this task is to transform a fine-granular Java syntax graph conforming to the JaMoPP metamodel [1] into a much simpler structure graph model that only contains statements and expressions that are neither structured nor subdivided any further. However, the original Java code of these statements and expressions should be reflected in the new elements' txt attribute. This model-to-text transformation is described in the next paragraph. Thereafter, the model-to-model transformation creating a structure graph from a JaMoPP model is described.

JaMoPP to Text. This model-to-text transformation is implemented using FunnyQT's polymorphic function API. A polymorphic function is a function that is declared once, and then arbitrarily many implementations for concrete metamodel types can be added. When a polymorphic function is called, the actual implementation is determined similarly to the typical dispatch in object-oriented programming languages. If there is no implementation provided for the element's type or one of its supertypes, an exception is thrown.

\footnotetext{
${ }^{1}$ The FunnyQT homepage: https://github.com/jgralab/funnyqt

${ }^{2}$ This FunnyQT solution is available at https://github.com/tsdh/ttc-2013-flowgraphs and on SHARE (image TTC13::Ubuntu12LTS_TTC13::FunnyQT.vdi
}

Pieter Van Gorp, Louis M. Rose, Christian Krause (Eds.): Sixth Transformation Tool Contest (TTC 2013)

EPTCS 135, 2013, pp. 57-68 doi 10.4204/EPTCS.135.7 
The function stmt2str implements the model-to-text transformation required for solving task 1. It is declared as follows.

(declare-polyfn stmt2str [elem])

declare-polyfn declares a new polymorphic function. Its name is stmt2str, and it receives exactly one parameter elem. Its task is to create a string representation matching the concrete Java syntax for the provided JaMoPP model element.

After the polymorphic function has been declared, implementations for concrete metamodel types can be added using defpolyfn. For example, this is the implementation for JaMoPP elements of type AssignmentExpression:

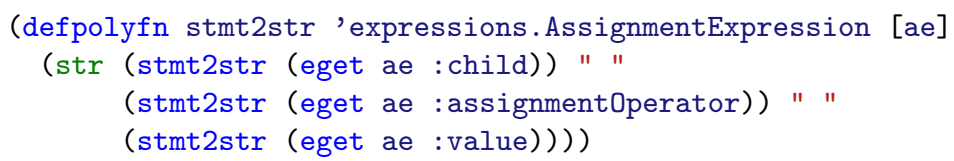

The child of the assignment expression is some variable, the assignmentOperator is one of $=,+=$, $-=, *=$, or $/=$, and value is an arbitrary expression. These three components are converted to strings using stmt2str again which are then concatenated.

All in all, the polymorphic stmt2str function consists of 22 implementations for various JaMoPP metamodel types accounting to a total of 107 lines of code. The complete model-to-text transformation is printed in Appendix A.

JaMoPP to Structure Graph. The JaMoPP-to-StructureGraph transformation is implemented using FunnyQT's model-to-model transformation API. This transformation also creates Var and Param objects as requested by task 3.1 .

The transformation starts by defining its name and input and output models.

(deftransformation java2flowgraph [ [in :emf] [out :emf]]

There could be arbitrarily many input and output models, and they could be of different kinds, e.g., a transformation could receive a JGraLab TGraph and some EMF model, and create an output EMF model. Here, it gets only the JaMoPP EMF input model which is bound to the variable in, and one single structure graph output model bound to out, which is also an EMF model.

In the body of such a transformation, arbitrarily many rules may be defined. The first one is the method2method rule shown in the next listing. The ${ }^{-}$:top metadata preceeding the rule name specifies that the rule is a top-level rule. Such rules are applied to all matching elements by the transformation itself, whereas non-top-level rules have to be called explicitly from a top-level rule (directly or indirectly).

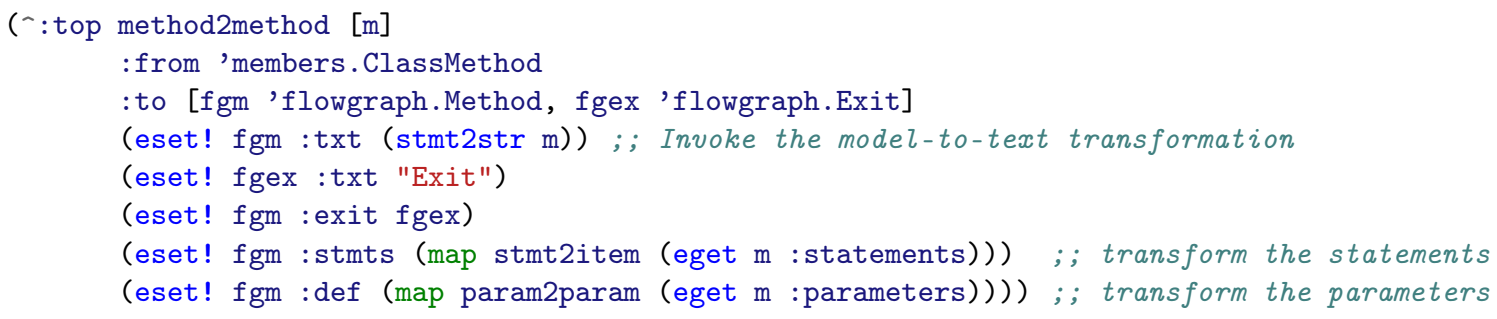

The rule receives a JaMoPP model element $m$. The :from clause dictates that m must be of type ClassMethod in order for the rule to be applicable. The : to clause declares the objects to be created. Here, for a given JaMoPP method, a corresponding flowgraph method and its exit object are created. 
The remainder of the rule is its body containing arbitrary code to set attributes and references. Here, the txt attribute of the new method and its exit are set, the former using the polymorphic stmt2str function discussed above. The method's stmts reference is set by applying another rule, stmt2item, to the statements of the JaMoPP method. Likewise, the method's parameters are transformed by mapping them to the param2param rule for setting the method's def reference.

Special kinds of rules are generalizing rules such as the one shown in the next listing.

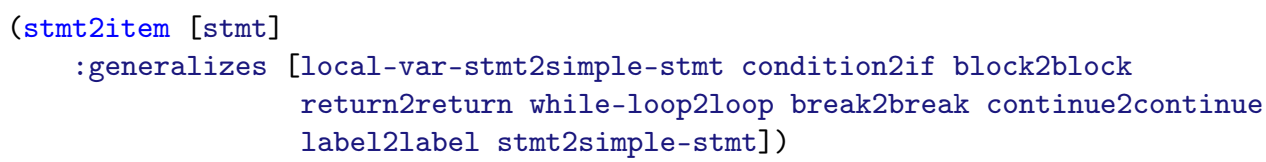

This concept is quite similar to mapping disjunction in QVT Operational Mappings. When this rule is called, the rules specified in the :generalizes vector are tried one after the other, and the first applicable one is applied, and its result is returned. Furthermore, a generalizing rule also combines the traceability mappings of all subrules.

The complete java2flowgraph model-to-model transformation consists of 15 rules with 93 lines of code in total. It is printed in Appendix B.

Task 2: Control Flow Analysis. The purpose of this task is to create cfNext links between FlowInstr elements in the flowgraph model created by the model-to-model transformation realizing task 1. Every such flow instruction should be connected to every other flow instruction that may be the next one in the program's control flow. This challenge has been tackled algorithmically using FunnyQT's plain quering and model manipulation APIs.

The algorithm uses a sequence of statements as intermediate representation to work on realizing a pre-order depth-first traversal with look-ahead through the method's statements. In the general case, every flow instruction in that sequence has to be connected with the immediately following flow instruction in the sequence. For various kinds of statements, special rules are needed. For example, when encountering a block in the sequence (which is no flow instruction), the block is replaced with its contents.

Since the next statement in the sequence might not be a flow instruction but some structured statement like a block, an if-statement, or a loop, there is a helper function cf-peek. It receives some element and returns either this element if it is a flow instruction, or otherwise the first flow instruction inside this element.

The function cf-synth synthesizing the control flow links using the algorithm sketched above is explained in the next four listings. It receives the sequence of statements $v$, the method's Exit node exit, the current loop's test expression (loop-expr), the statement following the current loop (loop-succ), and a map label-succ-map that assigns to each label reachable in the current scope the statement following the labeled statement. The exit parameter is used for handling return statements, and the last three parameters are used for handling break and continue statements. Initially, the function is called with v only containing the method, and exit bound to that method's exit. All other parameters are nil.

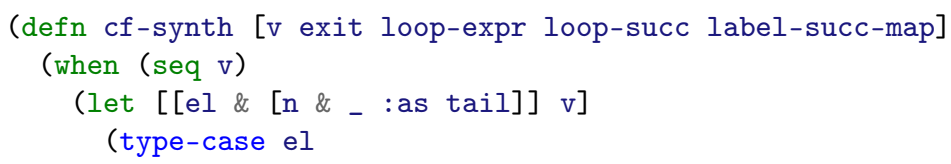

If the sequence $\mathrm{v}$ is not empty, its first element is bound to el, and its rest is bound to tail. Furthermore, the first element of the rest (i.e., the second element of the sequence) is bound to $\mathrm{n}$. 
After binding these elements, a type-case dispatches on el's metamodel type. For example, if the element is a method, a control flow link to that method's first flow instruction is created, and the function recurses with the method's statements (recur is an explicit tail-recursive call).

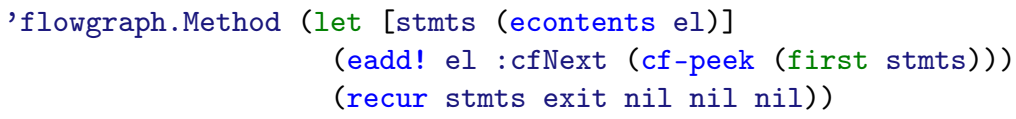

If the current element is a label, the function recurses with that label's statement prepended to the tail of the sequence. A mapping from this label to its following statement is added to the label-succ-map. This statement's first flow instruction is where the control flow continues when breaking to this label.

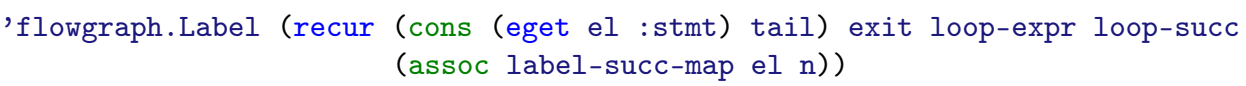

If the current element is a break statement, two cases have to be distinguished. If the break is labeled, a control flow link is added to the first flow instruction of the statement following the label which can be looked up in the label-succ-map. If the break is not labled, a control flow link is added to the first flow instruction in the statement following the surrounding loop which is bound to loop-succ.

In any case, the function recurses with the tail of the sequence keeping all other parameters as-is.

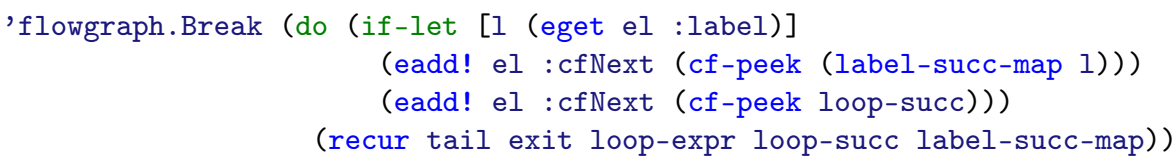

There are similar cases for handling objects of the other metamodel types. The complete control flow transformation consists of 57 lines of code and is printed in Appendix C.

Task 3: Data Flow Analysis. The purpose of this task is to create dfNext links between FlowInst elements where the target element is a control flow successor of the source element, the target element uses (reads) a variable that was defined (written) by the source element, and the variable has not been rewritten in between. This definition has been implemented exactly as stated here, because although it's not the most efficient algorithm for the task, it is very clear and concise.

The function $f$ ind-nearest-definers receives a flow instruction $f i$ and a variable uv used by it, and it returns a vector of the nearest control flow predecessors that define that variable.

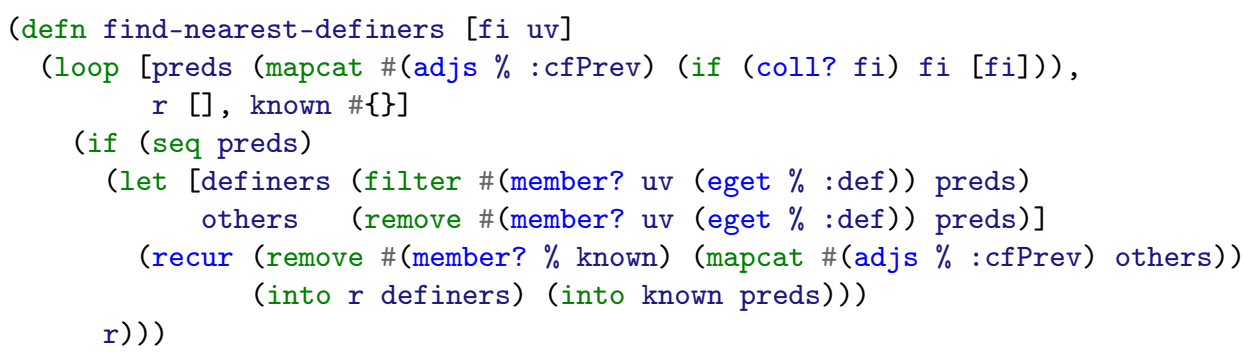

In Clojure, loop and recur implement a local tail-recursion, that is, inside a loop a recur form recurses not to the surrounding function but to the surrounding loop. Initially, preds is bound to the immediate control flow predecessors of $f i$, the result variable $r$ is bound to the empty vector, and known is bound to the empty set.

If there are no predecessors, the result $r$ is returned (the else-branch of the if). If there are control flow predecessors, those are sorted into definers and others, i.e., flow instructions that write to uv, and flow instructions that do not write to uv, respectively. 
Then it is recursed to the surrounding loop. preds is rebound to those control flow predecessors of others that aren't already known in order not to recurse infinitely in case of control flow cycles, the result vector $r$ is rebound to the current $r$ value plus the new definers, and known is rebound to the union of the current known value and the current preds.

The main function of this task simply uses this function to find the nearest definers of all flow instructions and their used variables and creates dfNext links.

The complete data flow transformation consists of 19 lines of code and is printed in Appendix D

Task 4: Control and Data Flow Validation. The goal of task 4 is to enable offloading testing effort for the transformations solving tasks 1 to 3 to programmers knowing only Java by equipping them with some easy to use DSL. The next listing shows an example validation specification as provided by the FunnyQT solution.

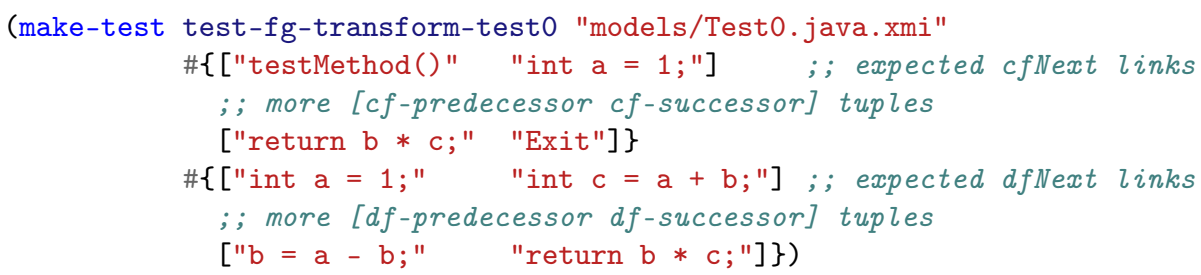

The FunnyQT solution uses Clojure's metaprogramming facilities to create an internal validation DSL. make-test is a macro. A macro is a function that will be called by the Clojure compiler at compiletime. It receives the unevaluated arguments given to it, that is, its parameters are bound to code. Clojure, like all Lisps, is homoiconic, meaning that code is represented using usual Clojure data structures, e.g., lists, vectors, symbols, literals, etc. Thus, the macro is able to transform the code provided to it using standard Clojure functions to some new bunch of code that takes its place. Here, make-test creates a unit test that loads the given XMI model and compares it against the expected control and data flow links.

The complete macro implementation and two complete validation specifications are printed in Appendix E.

\section{Evaluation}

In this section, the FunnyQT solution to the Flowgraphs case is evaluated according to the critera listed in the case description [2].

All four tasks have been solved, and the results of every task are complete and correct. The FunnyQT solution consists of 313 lines of code excluding comments and empty lines, making it the shortest of all provided solutions. It is also the solution with the best performance and has won the best efficiency award for this case. However, because FunnyQT is a Clojure API with a functional alignment, its understandability depends largely on a reader's prior knowledge about Clojure and functional programming.

\section{References}

[1] Florian Heidenreich, Jendrik Johannes, Mirko Seifert \& Christian Wende (2009): JaMoPP: The Java Model Parser and Printer. Technical Report TUD-FI09-10, Technische Universität Dresden, Fakultät Informatik. ftp://ftp.inf.tu-dresden.de/pub/berichte/tud09-10.pdf

[2] Tassilo Horn (2013): The TTC 2013 Flowgraphs Case. In Pieter Van Gorp, Louis Rose \& Christian Krause, editors: Sixth Transformation Tool Contest (TTC 2013), EPTCS, this volume. 


\section{A The complete JaMoPP-to-Text Transformation}

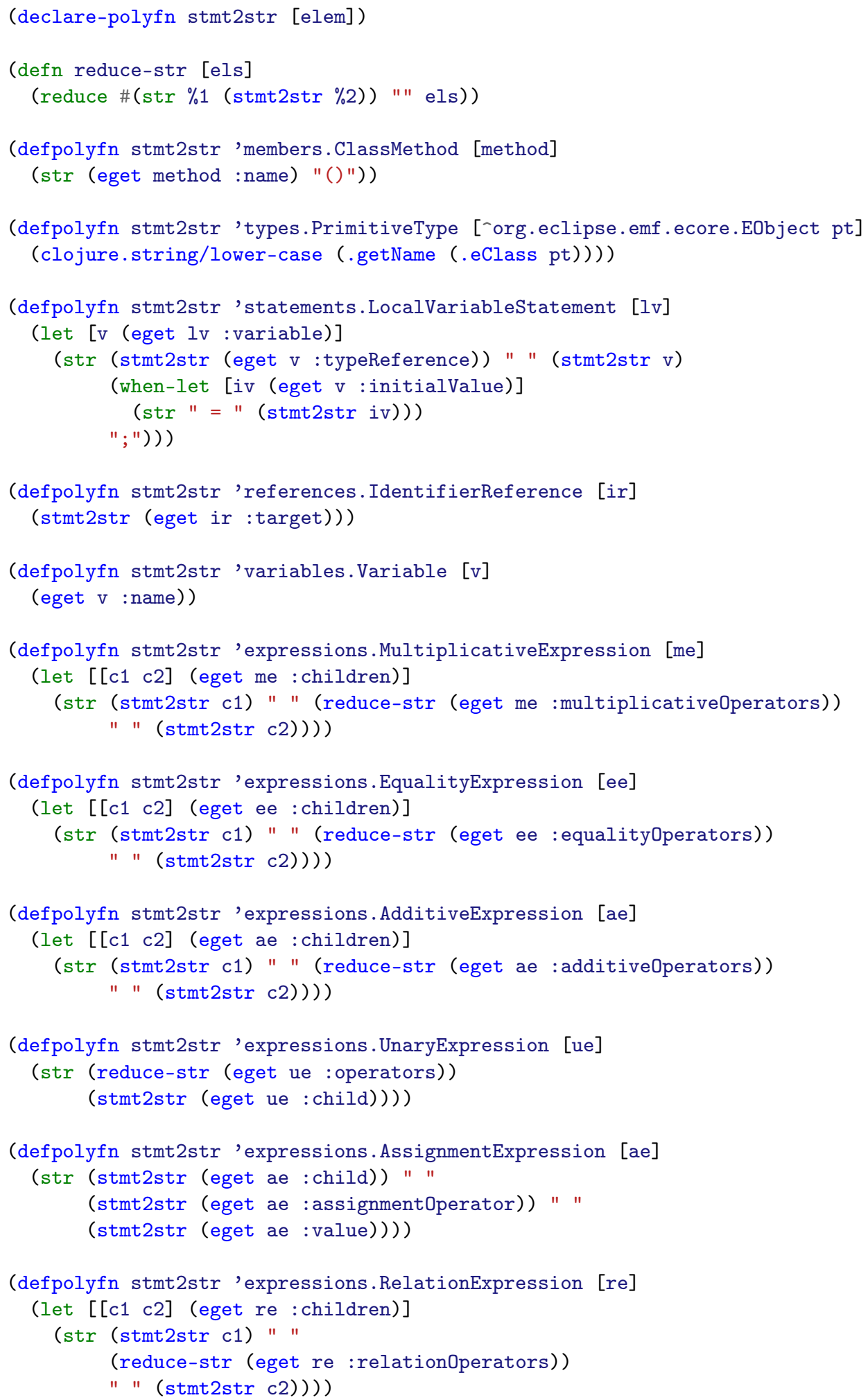




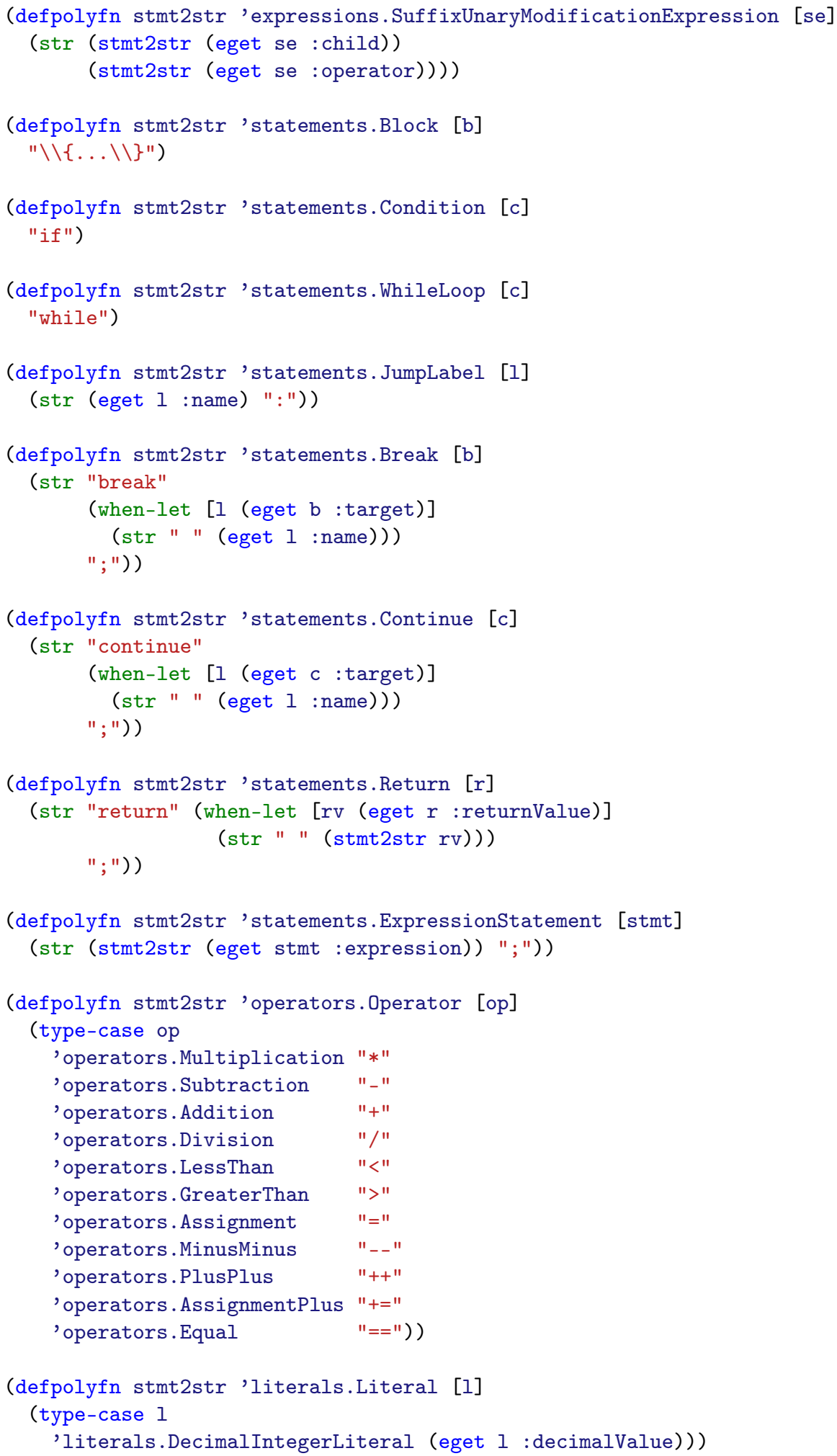




\section{B The complete JaMoPP-to-StructureGraph Transformation}

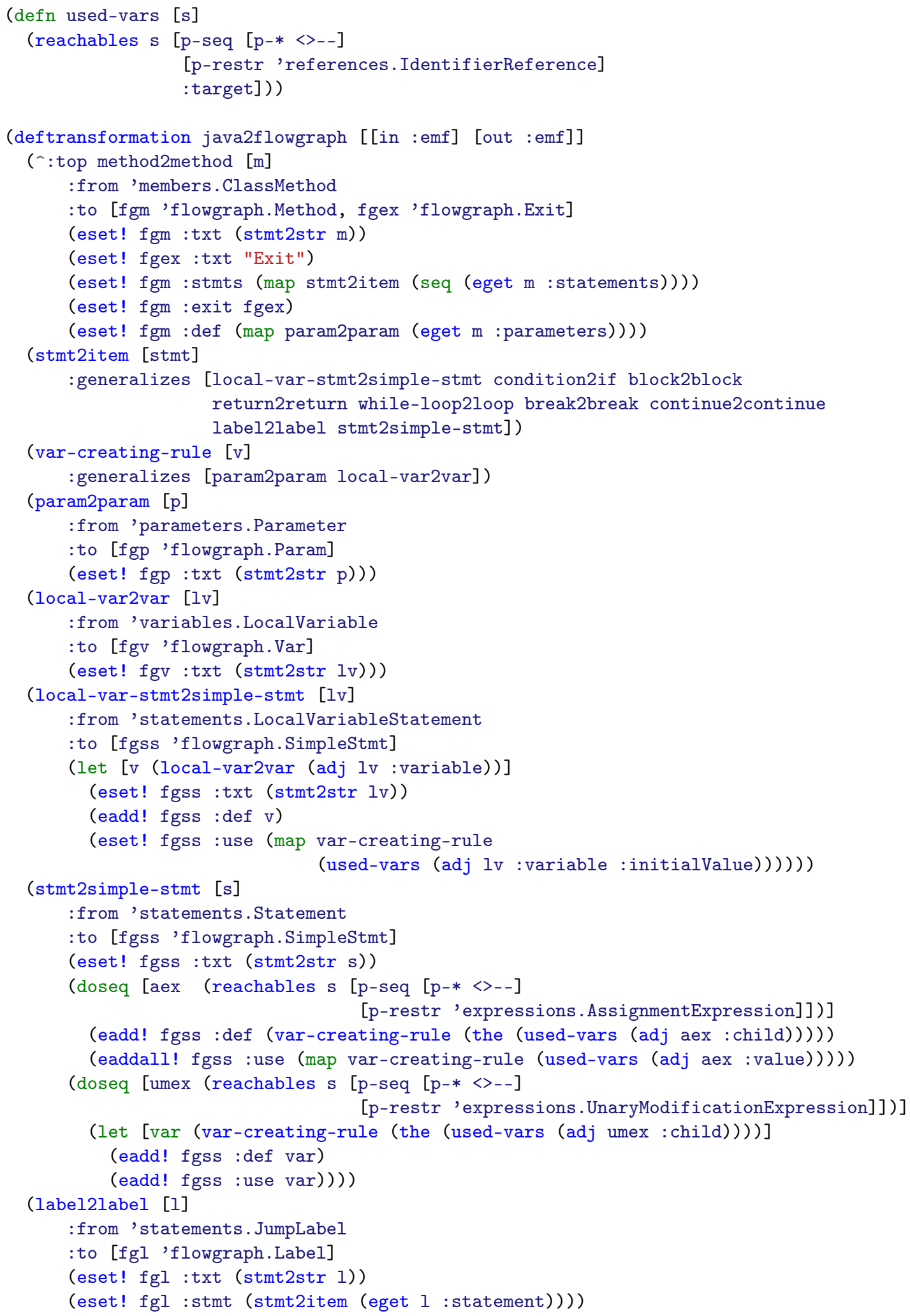




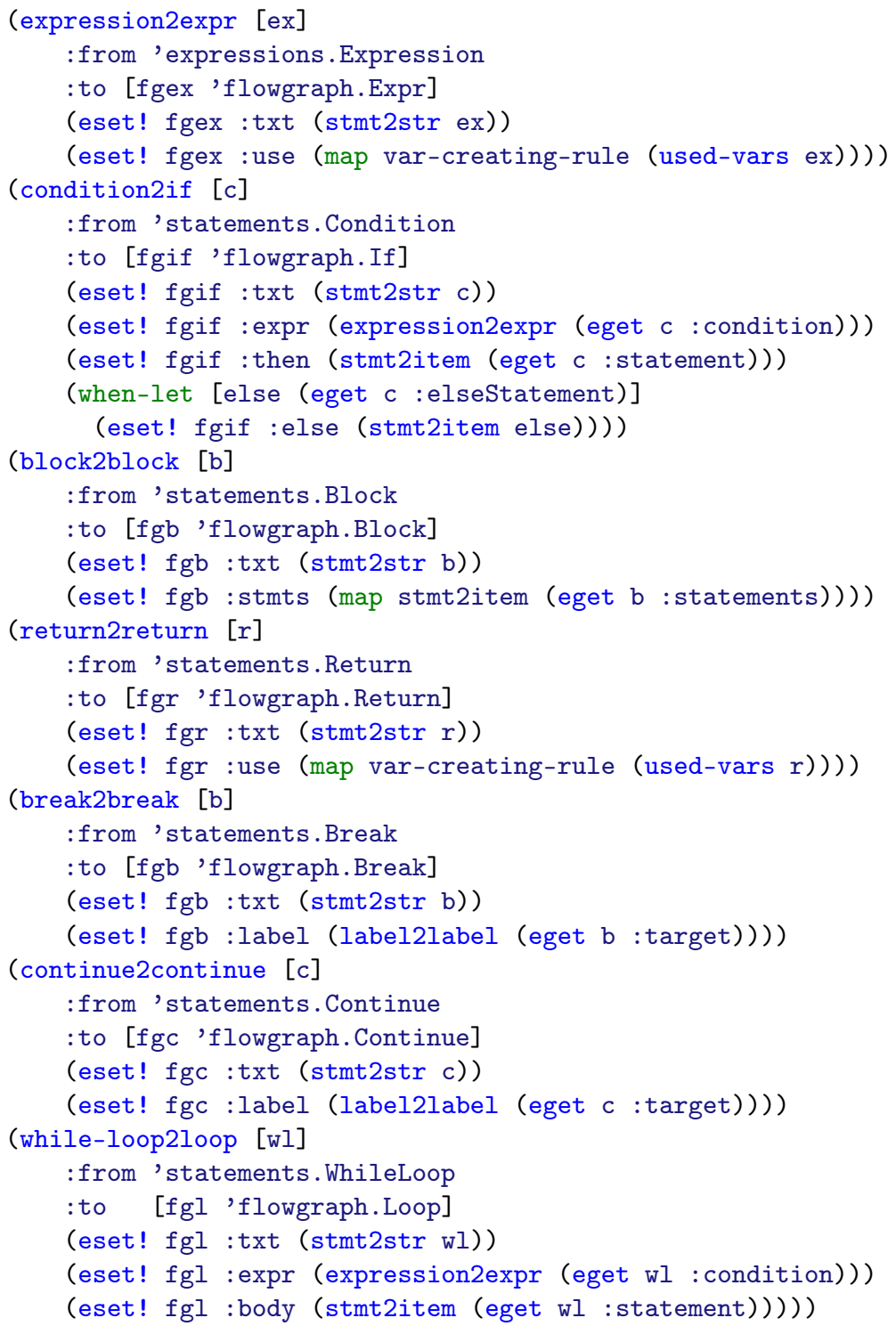

\section{The complete Control Flow Transformation}

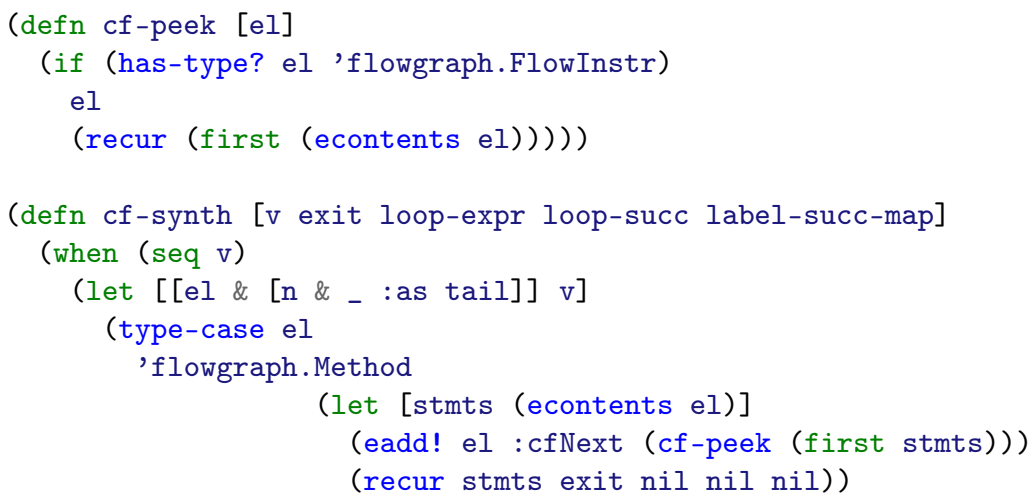




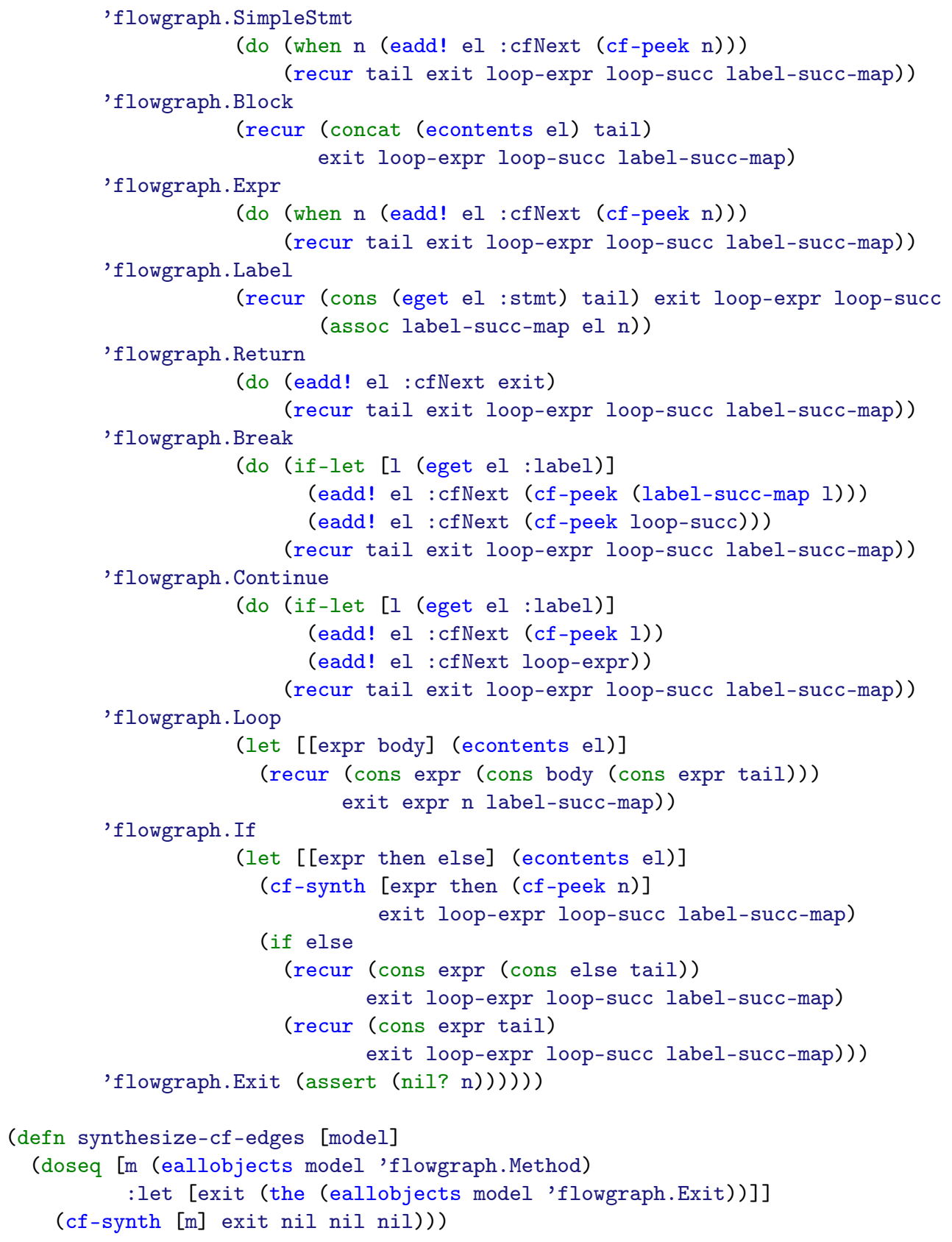

\section{The complete Data Flow Transformation}

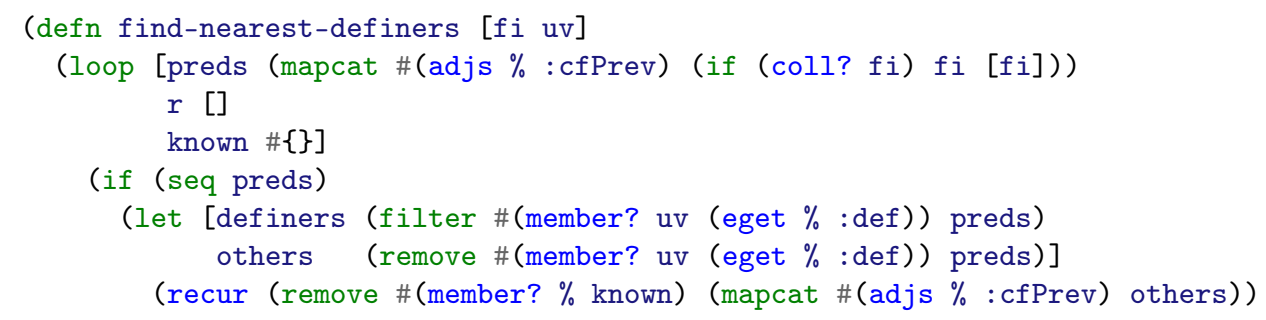




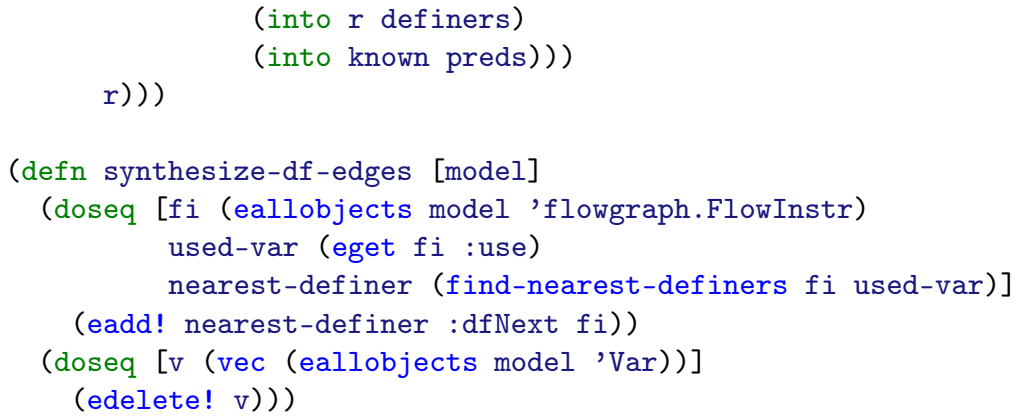

\section{E The complete Validation DSL Implementation}

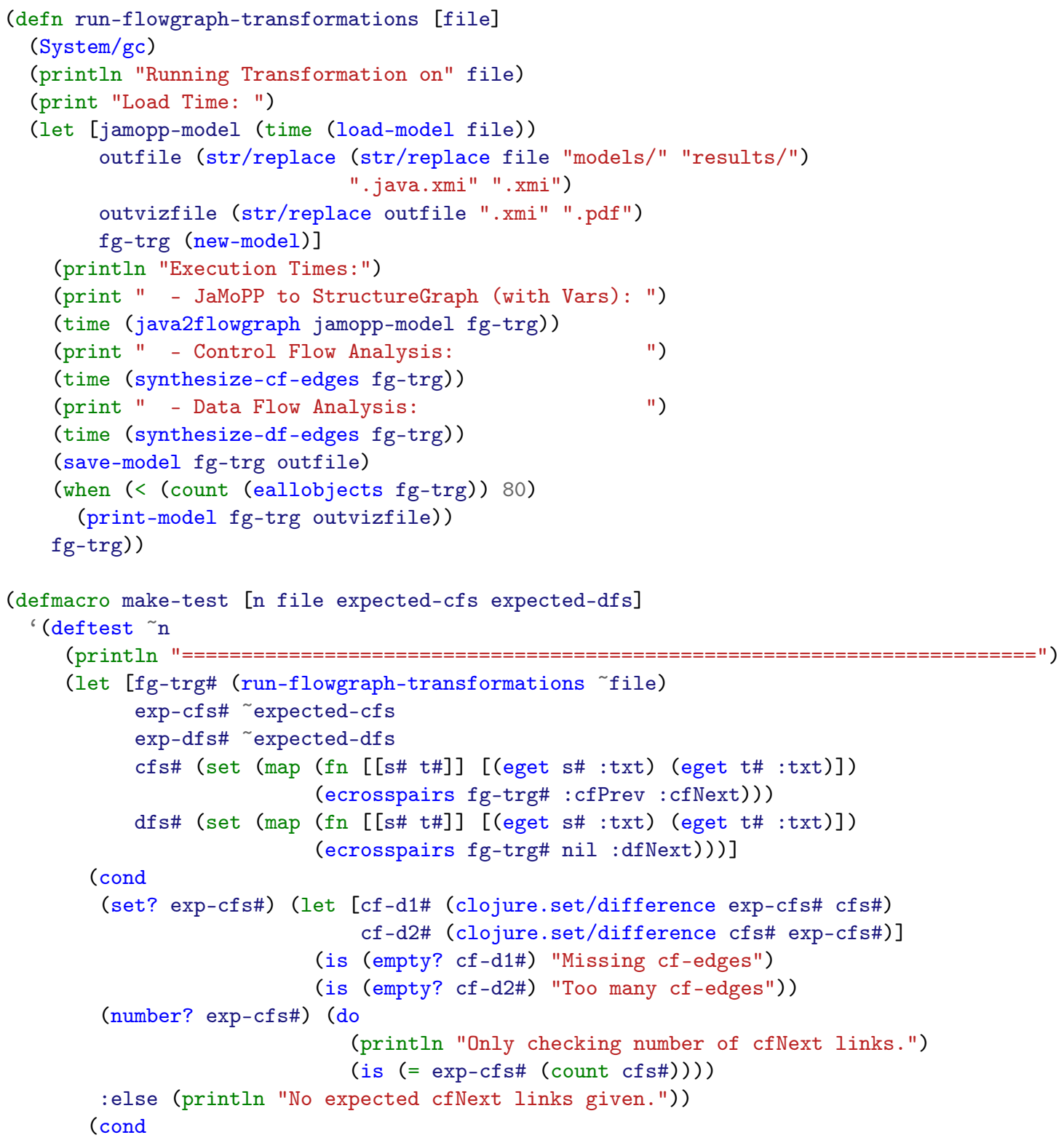




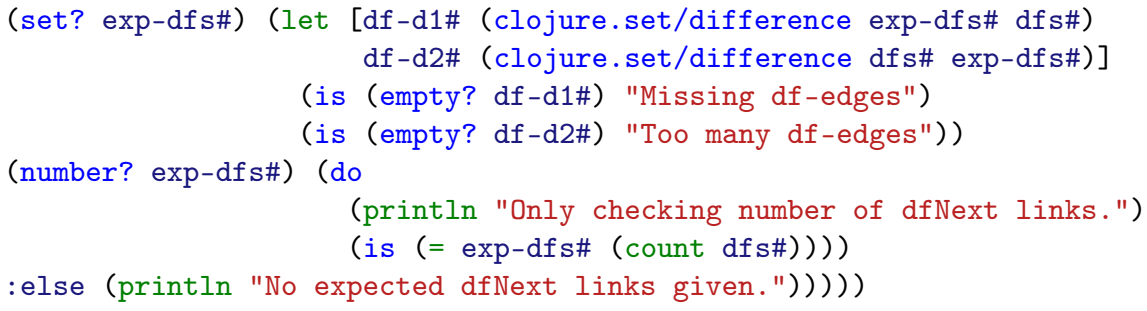

\section{E.1 Two Example Validation Specifications}

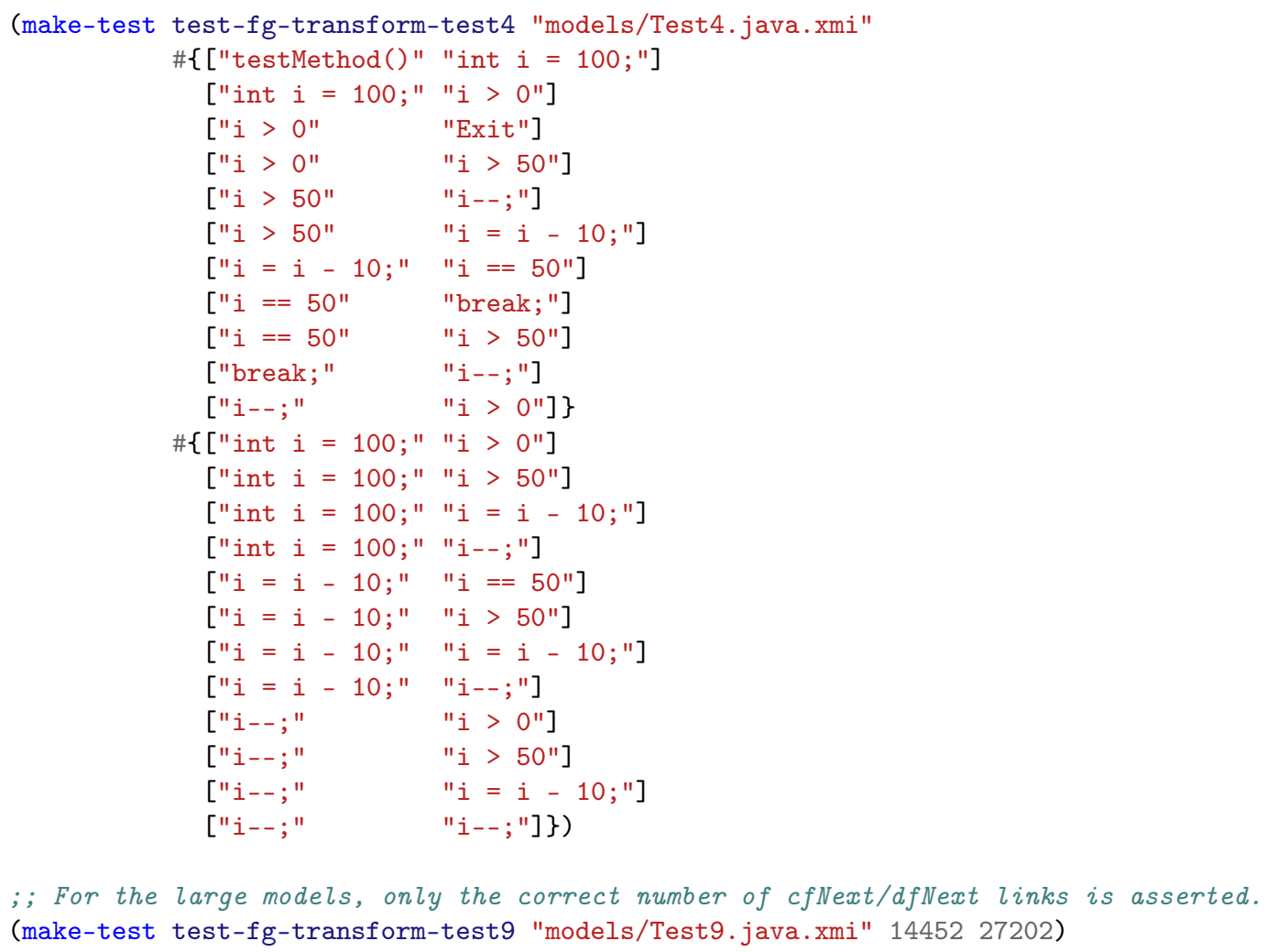

